\title{
Main pulmonary artery diameter from attenuation correction CT scans in cardiac SPECT accurately predicts pulmonary hypertension
}

\author{
Irene A. Burger, $M D,{ }^{a}$ Lars Husmann, $M D,{ }^{a}$ Bernhard A. Herzog, $M D,{ }^{a}$ \\ Ronny R. Buechel, MD, ${ }^{a}$ Aju P. Pazhenkottil, MD, ${ }^{a}$ Jelena R. Ghadri, MD, ${ }^{a}$ \\ Rene N. Nkoulou, MD, ${ }^{a}$ Rolf Jenni, MD, ${ }^{a}$ Erich W. Russi, MD, ${ }^{b}$ and \\ Philipp A. Kaufmann, $M^{\mathrm{a}, \mathrm{c}, \mathrm{d}}$
}

Objectives. To establish the value of the main pulmonary artery (MPA) diameter assessed from unenhanced computer tomography (CT) scans used for attenuation correction (AC) of single-photon emission computed tomography (SPECT) myocardial perfusion imaging (MPI) to predict pulmonary hypertension (PHT).

Background. In contrast-enhanced chest CT scans an MPA diameter of $29 \mathrm{~mm}$ or greater is an established predictor of PHT. However, it is unknown, whether measurements from an unenhanced CT scan for AC may be used as predictor of PHT.

Methods. 100 patients underwent SPECT MPI for assessment of coronary artery disease. PHT was defined as a right ventriculo-atrial gradient of $30 \mathrm{~mm} \mathrm{Hg}$ or greater by Doppler echocardiography. We compared MPA diameter from CT to SPECT findings (right ventricular hypertrophy/enlargement, septal wall motion abnormality/perfusion defect, and D-shape) to determine the best predictor of PHT.

Results. PHT was found in 37 patients. An MPA diameter of $30 \mathrm{~mm}$ or greater yielded a sensitivity, specificity, accuracy, positive, and negative predictive value of $78 \%, 91 \%, 86 \%$, $83 \%$, and $88 \%$, respectively. This yielded an area under the ROC curve of 0.85 .

Conclusions. MPA diameter from low-dose unenhanced multi-slice CT reliably predicts PHT, providing an important added clinical value from AC for SPECT MPI. (J Nucl Cardiol 2011;18:634-41.)

Key Words: Myocardial perfusion imaging • attenuation and scatter correction $\cdot$ SPECT

\section{INTRODUCTION}

Pulmonary hypertension (PHT) is an important predictor of an adverse outcome in patients with known or suspected coronary artery disease. ${ }^{1}$ Several findings of PHT have been reported in nuclear single-photon

From the Cardiac Imaging, ${ }^{\mathrm{a}}$ Pulmonology, ${ }^{\mathrm{b}}$ Nuclear Cardiology, ${ }^{\mathrm{d}}$ University Hospital Zurich, Zurich, Switzerland; and Zurich Center of Integrative Human Physiology (ZIHP), ${ }^{\mathrm{c}}$ University of Zurich, Zurich, Switzerland.

Received for publication Apr 1, 2010; final revision accepted Sep 22, 2010.

Reprint requests: Philipp A. Kaufmann, MD, Nuclear Cardiology, University Hospital Zurich, Ramistrasse 100, NUK C 42, 8091 Zurich, Switzerland; pak@usz.ch.

$1071-3581 / \$ 34.00$

Copyright (c) 2011 American Society of Nuclear Cardiology.

doi:10.1007/s12350-011-9413-9 emission computed tomography (SPECT) myocardial perfusion imaging (MPI), such as flattening of the interventricular septum (D-shaped left ventricle), septal wall motion abnormalities/perfusion defect, and right ventricular (RV) enlargement or hypertrophy. ${ }^{2,3}$ Attenuation correction (AC) of MPI with x-ray-derived attenuation maps obtained from a single-slice computer tomography scanner (CT) device has been introduced in the last years. ${ }^{4}$ However, transmission scans from single-slice CT scanners do not allow reliable interpretation of anatomic details. Recently, use of a high-end standalone CT scanner has been proposed to obtain AC maps for MPI. ${ }^{5}$ These low-dose data sets from a 64-slice scanner yield an image quality allowing anatomic evaluation.

While contrast-enhanced chest CT scans provide reliable signs of PHT such as enlarged main pulmonary artery (MPA) diameter, ${ }^{6}$ expanded RV outflow tract, and 
augmented distensibility of the MPA, ${ }^{7}$ as well as increased ratio of MPA to aortic diameter, ${ }^{8}$ the accuracy of unenhanced low-dose CT to reliably detect PHT from MPA diameter is not yet well validated.

The aim of this study was to identify the cut-off MPA diameter best predicting PHT from low-dose unenhanced multi-slice CT for AC of MPI.

\section{MATERIALS AND METHODS}

\section{Patients}

We retrospectively screened 183 consecutive patients referred for coronary artery disease assessment with SPECT MPI, until 100 patients were enrolled. To compare our results with the clinical gold standard for PHT and to have a study population with a high prevalence of PHT we applied the following inclusion criteria: first, transthoracic echocardiography within 6 months from SPECT MPI, documenting the right ventriculo-atrial (RV/RA) gradient in $\mathrm{mm} \mathrm{Hg}$. Second, a known or suspected pulmonary pathology such as bronchial carcinoma, dyspnea, PHT, chronic pulmonary embolism, chronic obstructive pulmonary disease, or emphysema.

The study protocol was approved by the institutional review board and written informed consent was obtained.

\section{SPECT MPI Acquisition}

SPECT MPI data acquisition was performed on a dualhead detector camera (Ventri, GE Healthcare), using a 1-day stress $(0.14 \mathrm{mg} / \mathrm{kg} / \mathrm{min}$ adenosine i.v. over 6 minutes, or dobutamine $10-40 \mu \mathrm{g} / \mathrm{kg} / \mathrm{min}) /$ rest protocol with a dose of approximately 300 and $900 \mathrm{MBq}$ of ${ }^{99 \mathrm{~m}} \mathrm{Tc}$ tetrofosmin, respectively, as previously reported. ${ }^{9}$ In brief, we acquired at $3^{\circ}$ rotation per stop, $180^{\circ}$ each head, and $25 \mathrm{~s}$ per projection, with 16 frames per R-R cycle, according to the procedural guidelines of the European Association of Nuclear in Medicine. ${ }^{10}$ For all patients, the summed nongated SPECT image set was reconstructed on a dedicated workstation (eNTEGRA or Xeleris; GE Healthcare). In addition, polar maps of wall motion (Figure 1) and perfusion (Figure 2) were produced
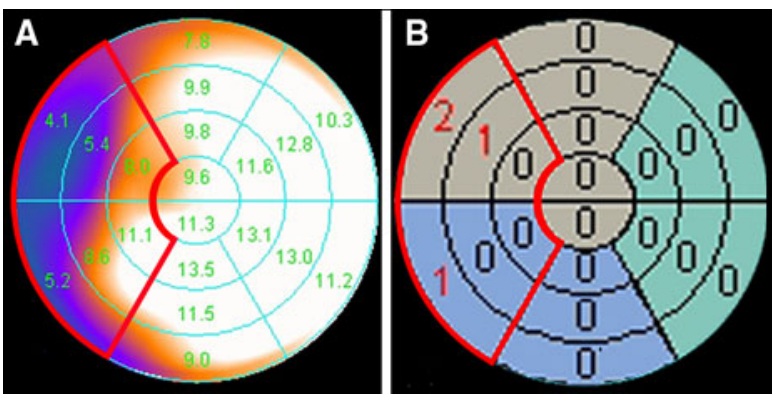

Figure 1. A 63-year-old man with severe chronic obstructive pneumopathy referred for cardiac risk assessment before lung transplantation. Polar plot image of rest wall motion (A), with corresponding semiquantitative scores $(\mathbf{B})$. The QGS scores of the six septal segments are adding up to a total score of 4 .
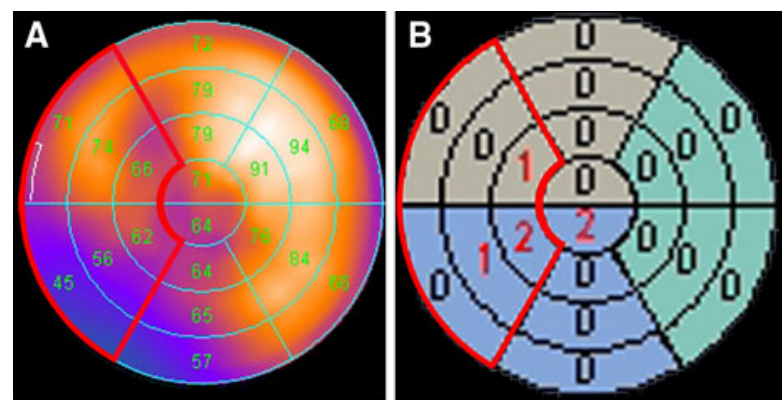

Figure 2. Same patient as in Figure 1. Polar plot image of stress perfusion filtered back projection image (A), with corresponding semiquantitative scores (B). The QPS scores of the six septal segments are adding up to a total score of 4 .

using a commercially available software solution (Cedars QGS/QPS; Cedars-Sinai Medical Centre). ${ }^{11}$

\section{SPECT MPI Interpretation}

SPECT MPI interpretation was visually performed by two nuclear cardiologists, who were blinded to the result from echocardiography and to the CT data. For each patient the longand short-axis slices were visually analyzed for two findings: flattening of the interventricular septum (D-shaped left ventricle) and RV enlargement appearing as large as or greater than the left ventricle. In addition, a quantitative analysis for RV hypertrophy was performed, using the peak pixel count of the RV and $\mathrm{LV}$ in the original filtered back projection SPECT rest images, to determine the $\mathrm{RV} / \mathrm{LV}$ ratio as previously reported. ${ }^{12} \mathrm{~A}$ ratio greater than 0.42 was regarded positive for RV hypertrophy according to results in humans by Williams et $\mathrm{al},{ }^{3}$ based on experimental animal data by Wackers et al. ${ }^{13}$ For quantitative assessment of septal wall motion (Figure 1B) and perfusion (Figure 2B) abnormality the respective QGS/QPS scores of the six septal segments were summed.

\section{CT Scanning}

All CT scans for AC were performed on a 64-slice CT on a LightSpeed VCT XT scanner (GE Healthcare) using prospective ECG-triggering (70\% of the R-R interval) and the following scanning parameters: gantry rotation time $350 \mathrm{~ms}$, $2.5 \mathrm{~mm}$ section thickness, $120 \mathrm{kV}$ tube voltage, $200 \mathrm{~mA}$ effective tube current, resulting in an estimated mean applied radiation dose of $1.1 \pm 0.1 \mathrm{mSv}$ (range $0.9-1.4 \mathrm{mSv}$ ). ${ }^{14}$

Scanning was performed from the tracheal bifurcation to the diaphragm, in inspiration and with elevated arms. CT images were reconstructed at $2.5-\mathrm{mm}$ slice thickness using a reconstruction algorithm with a $512 \times 512$ matrix. Reconstructed images were transferred to the external workstation for further analysis (AW 4.4, GE Healthcare).

\section{CT Image Analysis}

Two experienced radiologists, who were blinded to the results from echocardiography and the SPECT data, assessed the 


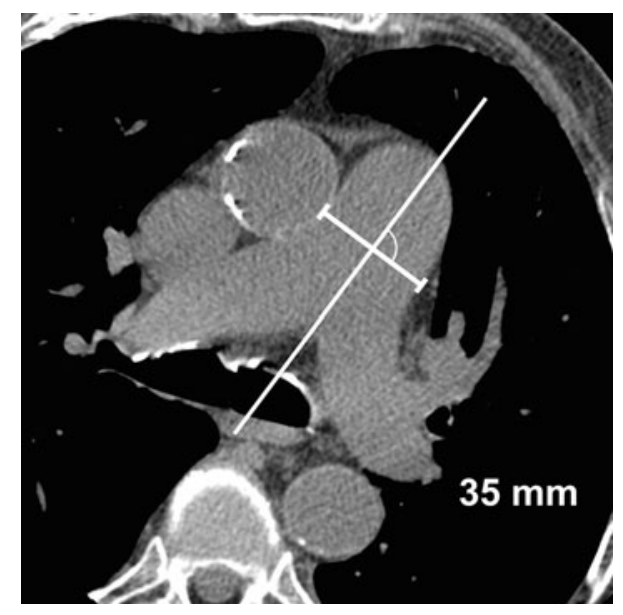

Figure 3. Main pulmonary artery (MPA) diameter is measured in standard axial slices in soft tissue window by assessing the widest diameter perpendicular to the long axis of the MPA.

maximum MPA diameter as follows: on standard axial sections in a soft tissue window (window width W400/level L40 Hounsfield units) the widest diameter perpendicular to the long axis of the MPA was measured with an electronic calliper (Figure 3). MPA diameter was divided by the calculated body surface area $(\sqrt{ }($ weight $(\mathrm{kg}) \times$ height $(\mathrm{cm})) / 60)$ to obtain indexed values.

\section{Echocardiography}

A two-dimensional transthoracic examination was performed in daily routine. The endsystolic tricuspid regurgitation jet velocity was used to determine the right ventriculo-atrial (RV/RA) gradient. PHT was defined as a value of $30 \mathrm{~mm} \mathrm{Hg}$ or greater, according to clinical standards. A RV/RA gradient between 30 and $39 \mathrm{~mm} \mathrm{Hg}$ was classified as mild PHT, a gradient between 40 and $59 \mathrm{~mm} \mathrm{Hg}$ as a moderate PHT and a value of $60 \mathrm{~mm} \mathrm{Hg}$ or greater as severe PHT. ${ }^{15}$

\section{Statistical Analysis}

The optimal cut-off point for predicting PHT was determined using a receiver operator characteristic (ROC) curve for the MPA diameter and the body surface corrected MPA diameter from unenhanced $\mathrm{CT}$, for the regional scores for septal stress perfusion defects as well as for the septal wall motion abnormalities. For the visually analyzed MPI SPECT signs such as D-shape and RV enlargement sensitivity, specificity, positive, and negative predictive values were calculated. In addition, all five SPECT MPI criteria for PHT (septal stress perfusion defects/wall motion abnormalities, RV hypertrophy/ enlargement and D-shape) and the MPA diameter were added up to a total score (0-6). ROC analysis was performed for these summed criteria. The optimal cut-off point for all ROC curves was determined using the Youden index ( $J$ value), calculated as the maximum of $J=\mathrm{SN}+\mathrm{SP}-1$, where $\mathrm{SN}$ is sensitivity and SP is specificity, for each cut-off volume. ${ }^{16}$ Areas under the ROC curve (AUC) were calculated and statistically compared with the Delong Delong Clarke-Pearson method ${ }^{17}$ using STATA software (STATA 10.1, Texas, USA).

Pearson's correlation coefficient and Bland-Altman analysis were used to determine the inter-observer agreement of the measured MPA diameter. A $P$ value of $<.05$ was considered to indicate statistical significance. Additional Bonferroni correction was performed, where appropriate. The inter-observer agreement of the visual analysis for D-shaped left ventricular deformation and enlarged right ventricle as well as for the measured MPA diameter was determined by kappa coefficient, with a $k$ value of 0.2-0.4 indicating a fair agreement, 0.41-0.6 a moderate agreement, 0.61-0.8 a substantial agreement, and 0.81-1.00 an almost perfect agreement, according to Landis and Koch. ${ }^{12}$ SPSS software (version 18.0, SPSS inc.) was used for statistical testing.

\section{RESULTS}

\section{Patient Characteristics}

Patient characteristics are listed in Table 1. From 100 enrolled patients with suspected or known pulmonary pathologies, 60 were referred for preoperative coronary artery disease assessment and in 40 patients the main reason for cardiac SPECT MPI was dyspnea. For further clinical information see Table 2. In 72 patients stress examination was performed with $0.14 \mathrm{mg} / \mathrm{kg} / \mathrm{min}$ adenosine i.v. without any complications. In 28 patients dobutamine stress was performed, due to known severe COPD.

In 2 patients a systemic lupus erythematosus was present, but none of them had an aneurysmal pulmonary artery. In 2 patients a $\alpha 1$-antitrypsin deficiency was known, one had no PHT, the second patient had a mild PHT (36 mm Hg pressure gradient) with moderate MPA dilatation of $32 \mathrm{~mm}$.

\section{CT and MPI Results}

AUC for MPA diameter as predictor of PHT was 0.85 (with a 95\% confidence interval 0.724-0.923, $P<.001$ ) with a resulting cut-off value of $30 \mathrm{~mm}$ using the YoudenIndex, which yielded a sensitivity, specificity, positive (PPV), and negative predictive value (NPV) and accuracy of $78 \%, 91 \%, 83 \%, 88 \%$, and $86 \%$, respectively. Correction of the MPA diameter for the calculated body surface area resulted in a slight decrease of the AUC (0.82), with a cut-off at $1.6 \mathrm{~cm} / \mathrm{m}^{2}$, therefore the uncorrected diameter was used for further analysis. Uncorrected MPA diameter cut-off at $30 \mathrm{~mm}$ with an AUC of 0.85 proved superior to semiquantitative scores for septal stress perfusion (AUC $0.61, P>.0002)$, wall motion abnormality $(P<.0001)$ and RV hypertrophy $(P=.037)$, although comparison of the latter versus MPA diameter fall short of statistical significance after correction according to Bonferroni (Figure 4). 
Table 1. Patient demographics

\begin{tabular}{ll}
\hline Number of patients & 100 \\
Age (mean \pm SD, years) & $65.2 \pm 11.2$ \\
Male gender & 64 \\
Weight (mean $\pm \mathrm{SD}, \mathrm{kg}$ ) & $75.6 \pm 16.7$ \\
Height (mean $\pm \mathrm{SD}, \mathrm{cm}$ ) & $168.6 \pm 9.5$ \\
BMI (mean $\pm \mathrm{SD}, \mathrm{kg} / \mathrm{m}^{2}$ ) & $26.4 \pm 5.3$ \\
Time interval between SPECT/CT & $0.36 \pm 2.5$ \\
$\quad$ and Echo (mean $\pm \mathrm{SD}$, months) &
\end{tabular}

$S D$, Standard deviation.

Table 2. Patient characteristics

\begin{tabular}{ll}
\hline $\begin{array}{l}\text { Number of patients with pulmonary } \\
\text { hypertension }\end{array}$ & 37 \\
Mild PHT & 19 \\
Moderate PHT & 12 \\
Severe PHT & 6 \\
Reason for CAD assessment & \\
Preoperative risk assessment & 60 \\
Resection of bronchial carcinoma & 24 \\
Wedge resection (other) & 8 \\
Lung transplantation & 10 \\
Liver surgery (in patients with suspected & 18 \\
$\quad$ PHT) & 40 \\
Dyspnea & 35 \\
Not further specified & 3 \\
Known PHT & 2 \\
Chronic pulmonary embolism & \\
Confirmed CAD by SPECT MPI & 26 \\
Total number of patients with a perfusion & 26 \\
$\quad$ defect & 18 (3) \\
Ischemia (Septal defect) & 20 \\
Scar & 12 \\
Both &
\end{tabular}

$C A D$, Coronary artery disease, $P H T$ : pulmonary hypertension. Mild PHT: RV/RA-gradient of $30-39.9 \mathrm{~mm} \mathrm{Hg}$.

Moderate PHT: RV/RA-gradient of $40-59.9 \mathrm{~mm} \mathrm{Hg}$.

Severe PHT: RV/RA-gradient of 60 or greater $\mathrm{mm} \mathrm{Hg}$.

The combined score of all 6 PHT signs (SPECT and CT) resulted in an AUC of $0.85(0.77-0.93, P<.001)$ with a cut-off at 2 positive criteria, which yielded a sensitivity, specificity, PPV, NPV, and accuracy of $86 \%$, $74 \%, 67 \%$, 90\%, and 79\%, respectively (Figure 4B). Thus, the AUC for the combination of SPECT and CT criteria was comparable to MPA diameter alone (0.85). However, specificity and accuracy were superior for MPA diameter alone (Table 3).

In 8 patients with documented mild PHT (RV/RA gradient between 30 and $39 \mathrm{~mm} \mathrm{Hg}$ ) MPA diameter was smaller than $3 \mathrm{~cm}$ classifying these as false negatives. No false negative results were found in patients with moderate or severe PHT. Only 6 patients had an RV/RA gradient over $60 \mathrm{~mm} \mathrm{Hg}$, indicating a severe PHT. The relationship between MPA diameter and PHT is not linear, as it flattens off at higher RV/RA gradients (Figure 5). Therefore, a reliable cut-off value to distinguish severe from non-severe PHT cannot be obtained.

The visual appreciation of the short- and long-axis SPECT MPI images of observer one resulted in 47 patients (38 patients for observer two) with a D-shaped left ventricular deformation. There was a moderate interobserver correlation with a kappa value of 0.533. 28 of the patients with diagnosed D-shape by observer one had a pulmonary hypertension; this resulted in a sensitivity, specificity, positive, and negative predictive value of $76 \%, 70 \%, 60 \%$, and $83 \%$, respectively. Relative RV enlargement was found only in 19 patients by observer one and in 25 patients by observer two, what conferred in a poor sensitivity $(37 \%)$ but high specificity $(92 \%)$ of observer one, with a positive and negative predictive value of $73 \%$ and $71 \%$, respectively, and a slightly higher sensitivity $(38 \%)$ but lower specificity $(82 \%)$ by observer two. The inter-observer agreement was fair with a kappa value of 0.362 . An overview of all results is given in Table 3.

Inter-observer analysis of MPA diameter assessment revealed an excellent correlation between the two observers $(r=0.94, P<.001)$ with narrow Bland-Altman limits of agreement ( -3.1 to $3.8 \mathrm{~mm}$ ) (Figure 6). This resulted in a $94 \%$ agreement of PHT diagnosis. There was no relation between a MPA diameter of $30 \mathrm{~mm}$ or greater and weight, height or BMI as reflected by the area under the ROC curve (AUC) of $0.47,0.53$, and 0.46 , respectively.

Two cases are illustrated in Figures 7 and 8.

\section{DISCUSSION}

Our results suggest that unenhanced CT scans as routinely performed for AC of SPECT MPI can be used to reliably predict PHT, since an MPA diameter of $30 \mathrm{~mm}$ or greater has a high sensitivity $(78 \%)$ and an excellent specificity (91\%), with a high inter-observer agreement $(r=0.94, P<.001)$. The established visual SPECT signs are less accurate, and have a moderate inter-observer agreement (kappa $=0.533$ for $\mathrm{D}$-shape and 0.362 for left ventricular enlargement). The best SPECT MPI finding is the RV hypertrophy, measured with the ratio of the peak pixel count RV:LV, with good specificity $(87 \%)$ but with a poor sensitivity (41\%), when using a cut-off at 0.42 .

Combining all five SPECT MPI criteria for PHT (septal perfusion defect, septal wall motion abnormality, 

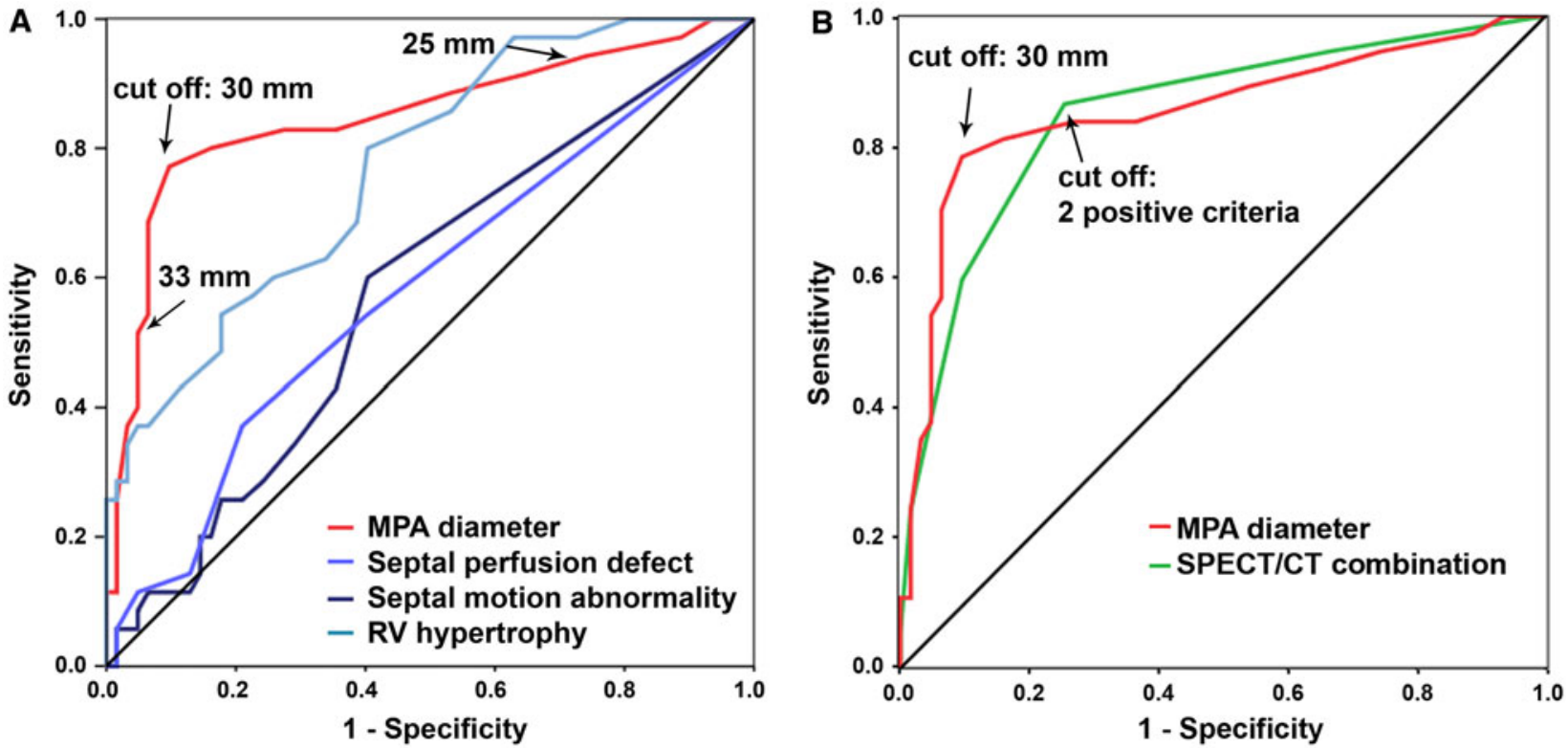

Figure 4. Receiver operating characteristic (ROC) curves are obtained in A for main pulmonary artery (MPA) diameter [red, which yields the largest area under the curve (0.85)], and for the semiquantitative scores for septal wall motion abnormalities (dark blue), septal stress perfusion defects (blue), and right ventricular hypertrophy (light blue). The resulting cut-off for MPA diameter is $30 \mathrm{~mm}$, yielding a sensitivity, specificity, positive, negative predictive value, and an accuracy of $78 \%, 91 \%, 83 \%, 88 \%$, and $86 \%$, respectively. B The ROC curve for MPA diameter (red) is compared to the ROC curve for the combined SPECT/CT score (green) (including septal stress perfusion defect and wall motion abnormality, RV hypertrophy, D-shape and RV enlargement, as well as the MPA diameter). The AUC for the combination of SPECT and CT criteria as well as for the MPA alone is identical (0.85), however specificity and accuracy are still higher for the MPA diameter.

Table 3. Predictors of PHT

\begin{tabular}{llllllll}
\hline \multicolumn{1}{c}{ Criteria } & $\begin{array}{c}\text { Septal } \\
\text { perfusion }\end{array}$ & $\begin{array}{c}\text { Septal } \\
\text { motion }\end{array}$ & $\begin{array}{c}\text { RV } \\
\text { hypertrophy }\end{array}$ & $\begin{array}{c}\text { D- } \\
\text { Shape }\end{array}$ & $\begin{array}{c}\text { RV } \\
\text { enlarged }\end{array}$ & $\begin{array}{c}\text { MPA } \\
\text { diameter }\end{array}$ & $\begin{array}{c}\text { SPECT/CT } \\
\text { combination }\end{array}$ \\
\hline AUC & $0.61^{*}$ & $0.59^{*}$ & $0.75^{*}$ & N/A & N/A & 0.85 & 0.85 \\
$95 \%$ CI & $0.492-0.729$ & $0.475-0.709$ & $0.661-0.850$ & N/A & N/A & $0.724-0.923$ & $0.768-0.931$ \\
$P$ value & .07 & .131 & .001 & N/A & N/A & $<.001$ & $<.001$ \\
Cut-off & 1 & 1 & 0.42 & N/A & N/A & 30 mm & 2 \\
Sensitivity (\%) & 59 & 56 & 40.5 & 76 & 35 & 78 & 86 \\
Specificity (\%) & 57 & 60 & 87.3 & 70 & 92 & 91 & 74 \\
PPV (\%) & 45 & 45 & 65.2 & 60 & 72 & 83 & 66 \\
NPV (\%) & 70 & 70 & 71.4 & 83 & 70 & 88 & 90 \\
Accuracy (\%) & 58 & 59 & 70 & 72 & 71 & 86 & 79 \\
\hline
\end{tabular}

$\mathrm{Cl}$, Confidence Interval.

${ }^{*} P<.05$, if compared with MPA diameter according to Delong Delong Clarke-Pearson.

D-shape, RV enlargement, and RV hypertrophy) with the MPA diameter from CT, yields an accuracy of 79\% (AUC 0.85 ) compared to $86 \%$ (AUC 0.85 ) of MPA diameter alone.
In the only previous study in which MPA was assessed from unenhanced CT images to evaluate PHT, a cut-off at $33.2 \mathrm{~mm}$ was determined yielding a specificity similar to our study but with a much lower 


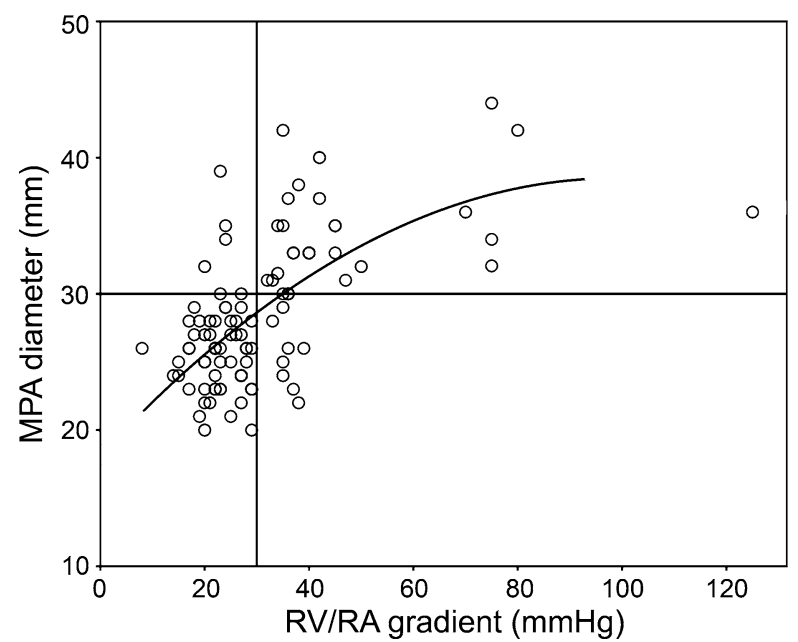

Figure 5. There was no linear correlation between pulmonary hypertension (represented by the RV/RA gradient) and MPA diameter, evidenced by the flattening of the curve for higher pressures. To determine a reliable cut-off to distinguish severe from non-severe PHT is in this study population not possible.

sensitivity $(58 \%) .{ }^{18}$ This was probably due-at least in part-to the fact that their slice thickness $(10 \mathrm{~mm})$ allowed less accurate measurements of anatomic structures than our CT data with a slice thickness of $2.5 \mathrm{~mm}$.

There is a large body of literature with contrast enhanced CT which has established an MPA diameter exceeding $29 \mathrm{~mm}$ as predictor of PHT. ${ }^{19}$ This value is slightly below our results, because contrast-enhanced CT provides the luminal diameter while in unenhanced
CT the distance of the outer vessel boundaries are measured.

The unenhanced CT scan of this study was performed to obtain attenuation maps for the SPECT MPI and not primarily for diagnostic purposes. Nevertheless, several reports on incidental findings with clinical relevance have stimulated a vivid controversy on whether a systematic readout of unenhanced CT for AC is mandatory for medico legal and ethical aspects or deleterious due to low specificity generating potentially unnecessary harms and costs of further examinations. It is in this setting that the present results are of great potential clinical importance as our MPA cut-off provides an excellent specificity combined with a large AUC to predict PHT, a clinically relevant finding with important implications for perioperative or long-term patient care directly affecting their outcome. Therefore, assessment of MPA diameter from unenhanced CT may be considered as a justifiable added value to SPECT MPI.

It may be perceived as a limitation of this study that echocardiography and CT examinations were not performed on the same day, as the pulmonary pressure may be subject to changes over time. However, the fact that despite this potential drawback the AUC was large strengthens our results and may support that MPA enlargement not only reflects acute increase in pulmonary pressure but also represents the result of longterm PHT. In addition, using echocardiography as gold standard for assessing PHT may be less accurate than
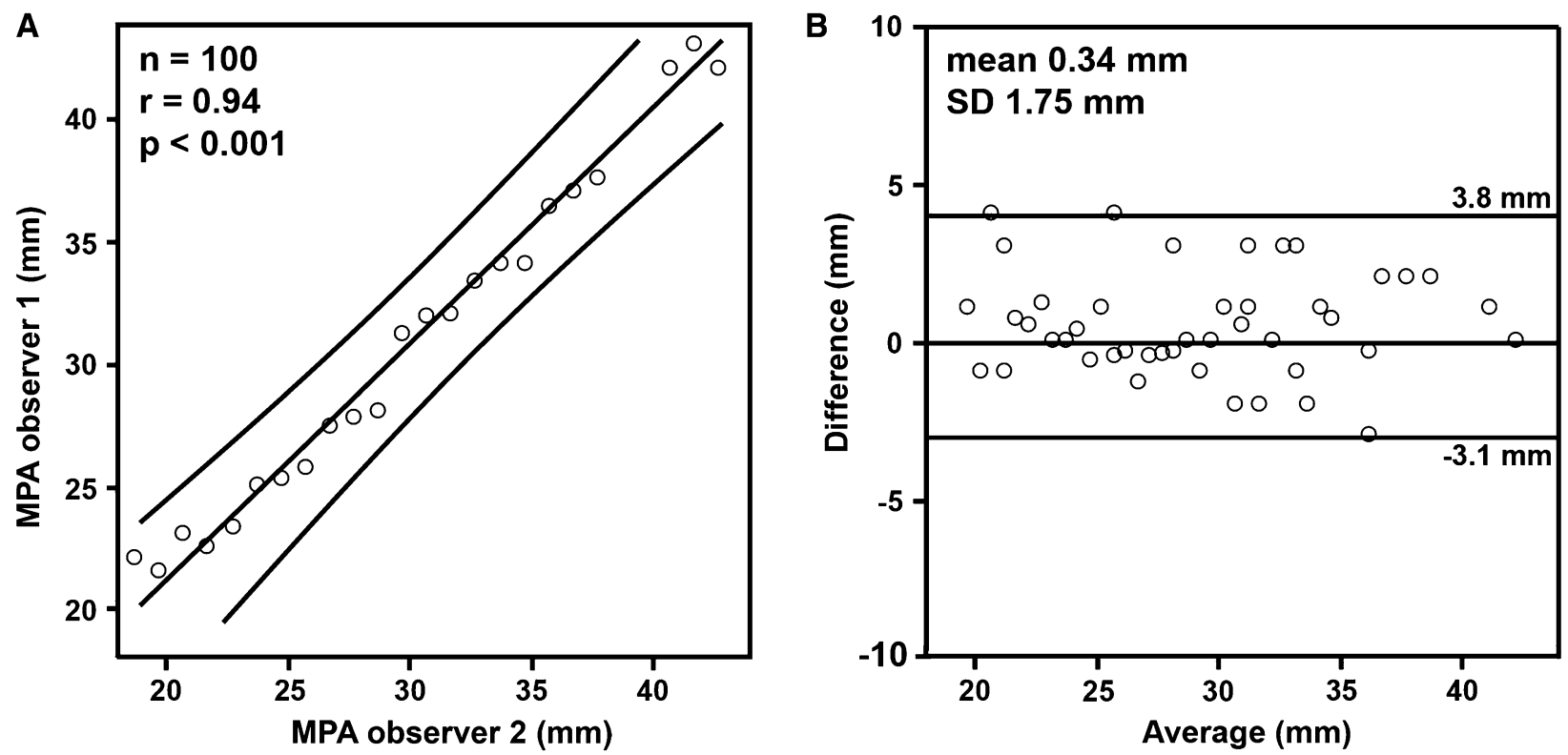

Figure 6. Inter-observer analysis of the main pulmonary artery diameter assessed by the two observers: Pearson correlation reveals an excellent correlation $(r=0.94, P<.001)(\mathbf{A})$, and BlandAltman analysis narrow limits of agreement (mean $0.34 \mathrm{~mm}$, SD $1.8 \mathrm{~mm})(\mathbf{B})$. 

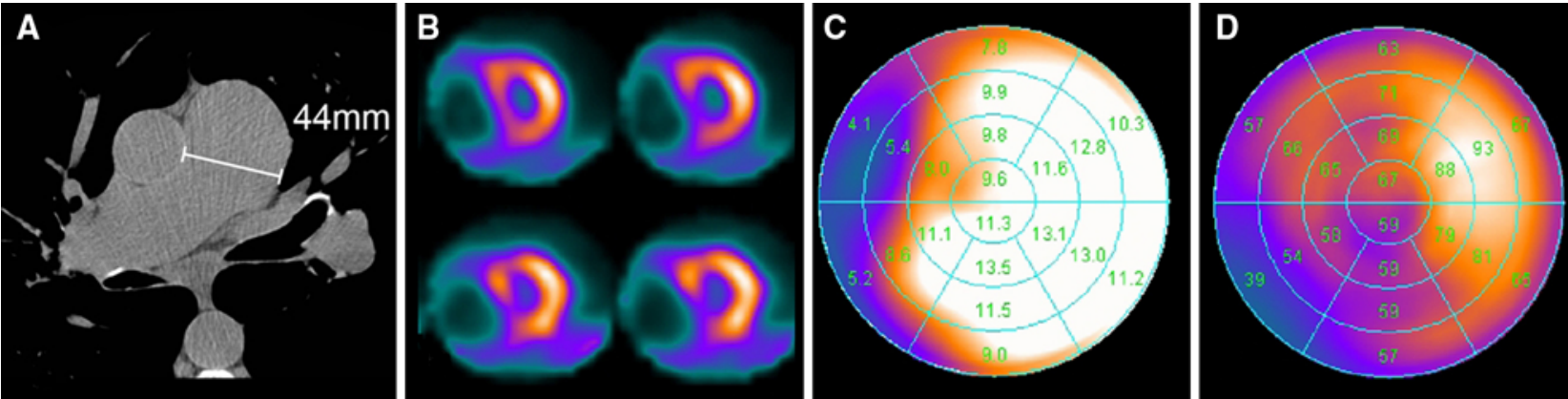

Figure 7. Same patient as in Figure 1. Echocardiography 2 weeks before SPECT MPI showed an $\mathrm{RA} / \mathrm{RV}$ gradient of $75 \mathrm{~mm} \mathrm{Hg}$. As in this case, $35 \%$ of patients with pulmonary hypertension documented by echocardiography reveal an enlarged main pulmonary artery (A) and typical SPECT MPI findings such as D-shaped left ventricle in axial short axis slices (B), wall motion abnormalities (C), and perfusion defect in filtered back projection stress perfusion (D).
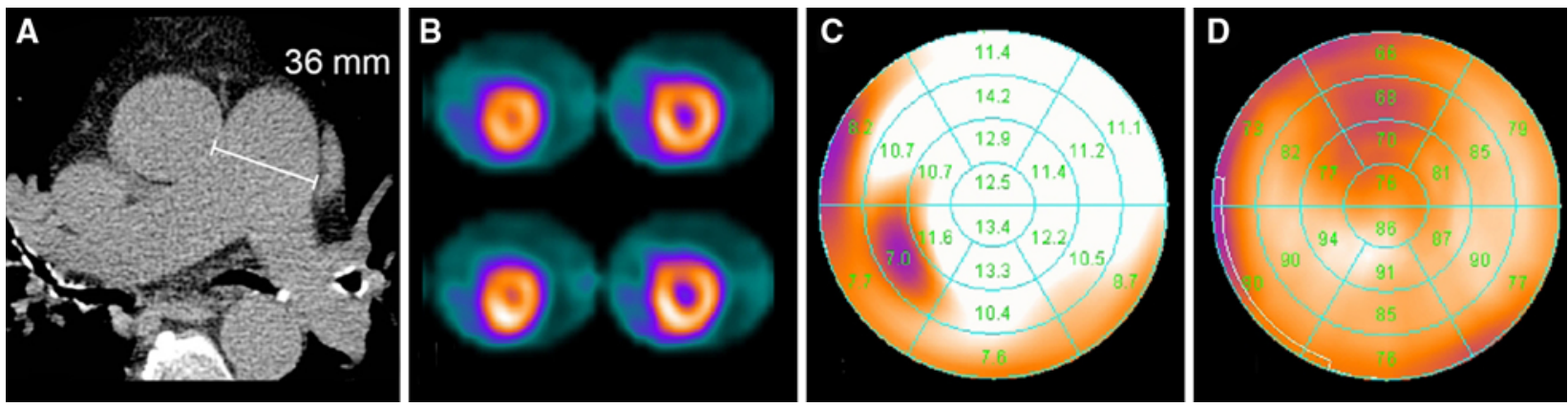

Figure 8. A 66-year-old woman with known chronic obstructive pneumopathy referred for cardiac risk assessment before wedge resection. Echocardiography 5 months before SPECT MPI showed an RA/RV gradient of $40 \mathrm{~mm} \mathrm{Hg}$. As in this case, $43 \%$ of patients with pulmonary hypertension have an enlarged main pulmonary artery (A) but SPECT MPI signs are not conclusive or negative [short axis slices $(\mathbf{B})$, wall motion rest $(\mathbf{C})$, and filtered back projection stress perfusion $(\mathbf{D})$ ].

invasive right heart catheterization. However, this corresponds to daily clinical routine, on which commonly decision making is based. Therefore, it appears justifiable to validate our CT-results against the clinical gold standard echocardiography.

Our results show that the sensitivity of the MPA diameter to predict PHT is not optimal (78\%), suggesting that unenhanced attenuation CT should not be recommended as a first line screening tool for PHT.

Another potential limitation is that the assessment of the RV/LV ratio in this study was assessed by use of the average counts in the area with the highest counts, while others have used the maximum count per pixel in this region. However, both methods have been shown to provide interchangeable and equally reproducible results. $^{12}$

Furthermore, the presence of collagen disorders in some of our patients may potentially hamper the generalization of our results, because such patients would be prone to early MPA enlargement at pulmonary pressures in the upper normal range. However, only in two of our patients a $\alpha 1$-antitrypsin deficiency was reported, of whom one was true positive and the other was true negative.

In addition, the image quality of our stand-alone CT scanner may not necessarily be matched by that obtained in Hybrid devices with low end CT scanners.

Finally, the prevalence of pulmonary hypertension in this study population is higher than in the general population referred for SPECT MPI in most centers. However, this selection appears reasonable for such a validation study, to include a sufficient number of patients presenting with the tested pathology, to avoid an artificially high specificity. This would strengthen our results when applied to low prevalence populations. The principle of MPA diameter as a reliable predictor of 
PHT has been well established for contrast-enhanced CT in large patient cohorts. Although this study extends this to unenhanced low-dose CT the pilot nature limits its power. Therefore, the finding should be interpreted with caution before data from larger studies are available. This is particularly true given that in clinical practice the 4\% patients misclassified with possible PHT would likely be referred on having further testing such as echocardiography.

\section{CONCLUSION}

The non-contrast-enhanced CT scan can be used to detect PHT, while SPECT MPI parameters are less accurate in detecting PHT. Thus, in patients undergoing SPECT MPI with a CT attenuation scan; this measurement can be reliably performed and may provide useful information about the potential PHT without any additional scans.

\section{References}

1. Galie N, Torbicki A, Barst R, Dartevelle P, Haworth S, Higenbottam $\mathrm{T}$, et al. Guidelines on diagnosis and treatment of pulmonary arterial hypertension. The Task Force on Diagnosis and Treatment of Pulmonary Arterial Hypertension of the European Society of Cardiology. Eur Heart J 2004;25:2243-78.

2. Movahed MR, Hepner A, Lizotte P, Milne N. Flattening of the interventricular septum (D-shaped left ventricle) in addition to high right ventricular tracer uptake and increased right ventricular volume found on gated SPECT studies strongly correlates with right ventricular overload. J Nucl Cardiol 2005;12:428-34.

3. Williams KA, Schneider CM. Increased stress right ventricular activity on dual isotope perfusion SPECT: A sign of multivessel and/or left main coronary artery disease. J Am Coll Cardiol 1999; 34:420-7.

4. Masood Y, Liu YH, Depuey G, Taillefer R, Araujo LI, Allen S, et al. Clinical validation of SPECT attenuation correction using $\mathrm{x}$-ray computed tomography-derived attenuation maps: Multicenter clinical trial with angiographic correlation. J Nucl Cardiol 2005; 12:676-86.

5. Schepis T, Gaemperli O, Koepfli P, Ruegg C, Burger C, Leschka $\mathrm{S}$, et al. Use of coronary calcium score scans from stand-alone multislice computed tomography for attenuation correction of myocardial perfusion SPECT. Eur J Nucl Med Mol Imaging 2007;34:11-9.

6. Kuriyama K, Gamsu G, Stern RG, Cann CE, Herfkens RJ, Brundage BH. CT-determined pulmonary artery diameters in predicting pulmonary hypertension. Invest Radiol 1984;19:16-22.

7. Revel MP, Faivre JB, Remy-Jardin M, Delannoy-Deken V, Duhamel A, Remy J. Pulmonary hypertension: ECG-gated 64-section CT angiographic evaluation of new functional parameters as diagnostic criteria. Radiology 2009;250:558-66.

8. Ng CS, Wells AU, Padley SP. A CT sign of chronic pulmonary arterial hypertension: The ratio of main pulmonary artery to aortic diameter. J Thorac Imaging 1999;14:270-8.

9. Husmann L, Herzog BA, Gaemperli O, Tatsugami F, Burkhard N, Valenta I, et al. Diagnostic accuracy of computed tomography coronary angiography and evaluation of stress-only single-photon emission computed tomography/computed tomography hybrid imaging: Comparison of prospective electrocardiogram-triggering vs retrospective gating. Eur Heart J 2009;30:600-7.

10. Hesse B, Tagil K, Cuocolo A, Anagnostopoulos C, Bardies M, Bax $\mathrm{J}$, et al. EANM/ESC procedural guidelines for myocardial perfusion imaging in nuclear cardiology. Eur J Nucl Med Mol Imaging 2005;32:855-97.

11. Germano G, Kavanagh PB, Waechter P, Areeda J, Van Kriekinge S, Sharir T, et al. A new algorithm for the quantitation of myocardial perfusion SPECT I: Technical principles and reproducibility. J Nucl Med 2000;41:712-9.

12. Mannting F, Zabrodina YV, Dass C. Significance of increased right ventricular uptake on $99 \mathrm{mTc}$-sestamibi SPECT in patients with coronary artery disease. J Nucl Med 1999;40:889-94.

13. Wackers FJ, Klay JW, Laks H, Schnitzer J, Zaret BL, Geha AS. Pathophysiologic correlates of right ventricular thallium-201 uptake in a canine model. Circulation 1981;64:1256-64.

14. Husmann L, Herzog BA, Burger IA, Buechel RR, Pazhenkottil $\mathrm{AP}$, von Schulthess $\mathrm{P}$, et al. Usefulness of additional coronary calcium scoring in low-dose CT coronary angiography with prospective ECG-triggering: Impact on total effective radiation dose and diagnostic accuracy. Acad Radiol 2010;17:201-6.

15. Oh JK, Seward JB, Tajik J. The Echo Manual. Rochester, MN: Lippincott-Raven; 1999. p. 215-22.

16. Fluss R, Faraggi D, Reiser B. Estimation of the Youden Index and its associated cutoff point. Biom J 2005;47:458-72.

17. DeLong ER, DeLong DM, Clarke-Pearson DL. Comparing the areas under two or more correlated receiver operating characteristic curves: A nonparametric approach. Biometrics 1988;44:837-45.

18. Edwards PD, Bull RK, Coulden R. CT measurement of main pulmonary artery diameter. Br J Radiol 1998;71:1018-20.

19. Tan RT, Kuzo R, Goodman LR, Siegel R, Haasler GB, Presberg KW. Utility of CT scan evaluation for predicting pulmonary hypertension in patients with parenchymal lung disease Medical College of Wisconsin Lung Transplant Group. Chest 1998;113: 1250-6. 